Cinémas

Revue d'études cinématographiques

Journal of Film Studies

\title{
Index des noms
}

Volume 15, numéro 2-3, printemps 2005

Cinélekta 5

URI : https://id.erudit.org/iderudit/012332ar

Aller au sommaire du numéro

Éditeur(s)

Cinémas

ISSN

1181-6945 (imprimé)

1705-6500 (numérique)

Découvrir la revue

Citer ce document

(2005). Index des noms. Cinémas, 15(2-3), 228-235.

Ce document est protégé par la loi sur le droit d'auteur. L'utilisation des services d'Érudit (y compris la reproduction) est assujettie à sa politique d'utilisation que vous pouvez consulter en ligne.

https://apropos.erudit.org/fr/usagers/politique-dutilisation/
Cet article est diffusé et préservé par Érudit.

Érudit est un consortium interuniversitaire sans but lucratif composé de l'Université de Montréal, l'Université Laval et l'Université du Québec à Montréal. Il a pour mission la promotion et la valorisation de la recherche. https://www.erudit.org/fr/ 


\section{Index des noms}

AGEE James

"Common People with Common

Feelings" : Pauline Kael, James

Agee, and the Public Sphere of

Popular Film Criticism

Vol. 6, n ${ }^{\text {os }}$ 2/3 : 113 d

AITKEN William Maxwell

William Maxwell Aitken, père

tout-puissant du cinéma canadien

Vol. 10, n ${ }^{\circ} 1: 13 \sim \mathrm{p}$

AKERMAN Chantal

Je, tu, il, elle

Vol. 8, $\mathrm{n}^{\circ}$ 3: 121 z

ALLIO René

Camisards (Les)

Vol. 5, $\mathrm{n}^{\text {os }} 1 / 2: 21 \sim \mathrm{d}$

ALTMAN Robert

Nashville

Vol. 1, no 3: 103 - d

AMELIO Gianni

Enfants volés (Les)

Vol. 12, $\mathrm{n}^{\circ} 1: 53 \sim \mathrm{z}$

Lamerica

Vol. 12, n $1: 56 \sim \mathrm{z}$

Le paysage de l'expérience

Vol. 12, $\mathrm{n}^{\circ} 1: 49 \sim \mathrm{z}$

Mon frère

Vol. 12, $\mathrm{n}^{\circ} 1:$ 64 z

ANGELOPOULOS Théo

Paysage dans le brouillard

Vol. 4, n $3: 31 \sim \mathrm{t}$

ANN Hui

Chant d'exil

Vol. 3, n ${ }^{\text {os }}$ 2/3 : 134 p

Princesse Parfum

Vol. 3, $\mathrm{n}^{\text {os }} 2 / 3: 141 \sim \mathrm{z}$

Roman du livre et de l'épée (Le)

Vol. 3, $\mathrm{n}^{\text {os }} 2 / 3: 141 \sim \mathrm{z}$

\author{
ANTONIONI Michelangelo \\ Avventura (L') \\ Vol. 6, $\mathrm{n}^{\text {os }} 2 / 3$ : 45 d \\ Blow-Up \\ Vol. 5, n ${ }^{\circ}$ 3: 56 d \\ ARCAND Denys
}

Entre l'écho des voisins et celui des cousins. Les voix croisées de la réception critique des films de Denys Arcand aux États-Unis et en France

Vol. 7, n 3: 119 z

Déclin de l'empire américain (Le)

Vol. 7, n' 3: 123 z

De l'amour et des restes humains

Vol. 7, n 3 : 129 z

Jésus de Montréal

Vol. 7, n 3: 125 z

AVERY Tex

Vitesse et dématérialisation. Le

corps du toon chez Tex Avery

Vol. 7, $\mathrm{n}^{\text {os }} 1 / 2: 55 \sim \mathrm{z}$

BABENCO Hector

Baiser de la femme araignée (Le)

Vol. 10, n $1: 79 \sim \mathrm{z}$

BÉLANGER Fernand

Émotion dissonante (L')

Vol. 4, n ${ }^{\circ}$ : : 125 p

Passiflora

Vol. 4, n ${ }^{\circ}$ : : 125 p

BERGMAN Ingmar

Ingmar Bergman : fouiller le passé Vol. 9, n ${ }^{\text {os }} 2 / 3: 29 \sim \mathrm{z}$

BISSONNETTE Sophie

Amour... à quel prix? (L')

Vol. 1, $\mathrm{n}^{\text {os }} 1 / 2$ : 92 p

BRAULT Michel

Pour la suite du monde

Vol. 7, n 3 : 164 -t 
BRESSON Robert

Corps au cinéma: Keaton, Bresson, Cassavetes (Le) (Vincent Amiel)

Vol. 11, n ${ }^{\text {os }} 2 / 3: 309-1$

BROOK Peter

Moderato cantabile

Vol. 4, n ${ }^{\circ} 1: 89 \sim \mathrm{z}$

CAMERON James

Aliens, le retour

Vol. $7, \mathrm{n}^{\text {os }} 1 / 2: 121 \sim \mathrm{z}$

Terminator 2, le jugement dernier

Vol. 5, n 3: 131 z

\section{CAPRA Frank}

Italian and Irish Filmmakers in

America: Ford, Capra, Coppola and

Scorsese (Lee Lourdeaux)

Vol. 2, n $1: 170 \sim 1$

New York - Miami

Vol. $13, \mathrm{n}^{\text {os }} 1 / 2$ : 172 d

CARAX Leos

Boy Meets Girl

Vol. 5, n 3: 77 z

CASSAVETES John

Bal des vauriens (Le)

Vol. 13, n 3: 79 z

Corps au cinéma: Keaton, Bresson,

Cassavetes (Le) (Vincent Amiel)

Vol. 11, n ${ }^{\text {os }} 2 / 3: 309-1$

Husbands

Vol. 13, n 3: 79 z

CHABOT Jean

Voyage en Amérique avec un cheval emprunté

Vol. 1, n ${ }^{\text {os }} 1 / 2: 97 \sim p$

Vol. 1, n ${ }^{\text {os }} 1 / 2$ : 109 p

CHANG Sylvia

Passion

Vol. 3, $\mathrm{n}^{\text {os }}$ 2/3: 135 p

\section{CHEN Kaige}

Deux pôles yang du nouveau

cinéma chinois: Chen Kaige et

Zhang Yimou

Vol. 3, $\mathrm{n}^{\text {os }} 2 / 3: 103 \sim \mathrm{p}$

Roi des enfants (Le)

Vol. 3, $\mathrm{n}^{\text {os }} 2 / 3: 9 \sim \mathrm{z}$
CHIASSON Herménégilde

Grand Jack (Le)

Vol. $1, n^{\text {os }} 1 / 2: 95 \sim p$

CIMINO Michael

Sunchaser

Vol. 12, n $1: 105 \sim \mathrm{z}$

CISSÉ Souleymane

Enjeux interprétatifs de la féminité

dans l'écriture de Souleymane Cissé

Vol. 11, n ${ }^{\circ}$ 1: 61 z

CLÉMENT René

Bataille du rail (La)

Vol. 9, $\mathrm{n}^{\text {os }}$ 2/3: 210 d

COPPOLA Francis Ford

Italian and Irish Filmmakers in

America: Ford, Capra, Coppola and

Scorsese (Lee Lourdeaux)

Vol. 2, n $1:$ 170 1

COSTNER Kevin

Danse avec les loups

Vol. 8, $\mathrm{n}^{\text {os }} 1 / 2: 169 \sim \mathrm{z}$

Vol. 12, n ${ }^{\circ} 1: 105 \sim \mathrm{z}$

CRONENBERG David

Horreur intérieure: les films de

David Cronenberg (L') (Piers

Handling et Pierre Véronneau)

Vol. 2, $\mathrm{n}^{\text {os }} 2 / 3$ : 237 -

Mouche (La)

Vol. 4, n 2 : 153 x

Vidéodrome

Vol. 7, $\mathrm{n}^{\text {os }} 1 / 2: 73 \sim \mathrm{z}$

CURTIS Edward

In the Land of the Headhunters

Vol. 6, $\mathrm{n}^{\circ} 1: 33 \sim \mathrm{z}$

In the Land of the War Canoes

Vol. 6, n ${ }^{\circ} 1: 33 \sim \mathrm{z}$

DAO Mustapha

À nous la rue

Vol. 11, n $1: 31 \sim \mathrm{z}$

DELEUZE Gilles

Accords et faux raccords entre les conceptions du cinéma de Jean-Luc Godard et de Gilles Deleuze

Vol. 13, no 3 : 173 d 
Deleuze on Cinema (Ronald Bogue)

Vol. 14, n 1 : 207 -1

\section{DEMME Jonathan}

Silence des agneaux (Le)

Vol. 5, $\mathrm{n}^{\text {os }} 1 / 2$ : 91 d

DJEBAR Assia

Assia Djebar. Écrire, transgresser, résister (Jeanne-Marie Clerc)

Vol. 10, $n^{\text {os }} 2 / 3: 243-1$

\section{DUCHARME Réjean}

L'un(e) dort, l'autre pas : la scène de la veille dans les scénarios et quelques romans de Réjean

Ducharme

Vol. 5, $\mathrm{n}^{\text {os }} 1 / 2: 189 \sim \mathrm{z}$

DUGUAY Christian

Planète hurlante

Vol. 7, n $3: 152 \sim \mathrm{z}$

DURAS Marguerite

Césarée

Vol. 3, $\mathrm{n}^{\circ} 1: 115 \sim \mathrm{z}$

Écran de la passion. Une étude du cinéma de Marguerite Duras (L')

(Michelle Royer)

Vol. 8, $\mathrm{n}^{\circ} 3: 183-1$

India Song

Vol. 5, $\mathrm{n}^{\text {os }} 1 / 2: 123 \sim \mathrm{z}$

EISENSTEIN Sergueï

Comment éditer Eisenstein?

Problème de "Méthode» (extraits inédits)

Vol. 11, $\mathrm{n}^{\text {os }} 2 / 3: 27 \sim \mathrm{z}$

Eisenstein dans le texte - Notes au lecteur

Vol. 11, $\mathrm{n}^{\text {os }} 2 / 3: 9 \sim \mathrm{z}$

Inédit A.I. 28 [Attraction

intellectuelle 1928]

Vol. 11, n ${ }^{\text {os }} 2 / 3$ : 147 s

Intériorité/plasticité. La théorie de

la mise en scène de $S$. M. Eisenstein

Vol. $11, \mathrm{n}^{\text {os }} 2 / 3: 225 \sim \mathrm{z}$

Ivan le Terrible

Vol. $11, \mathrm{n}^{\text {os }} 2 / 3: 255 \sim \mathrm{z}$

Letter to Ivor Montagu

Vol. 11, n ${ }^{\text {os }} 2 / 3: 69 \sim$ s
Lettres à Léon Moussinac

Vol. 11, $\mathrm{n}^{\text {os }}$ 2/3: 161 s

Présentation: Pourquoi Eisenstein

"dans le texte»?

Vol. 11, $\mathrm{n}^{\text {os }} 2 / 3: 11 \sim \mathrm{z}$

"Rayon " et "Gnôle» [Essai de

définition de la carence idéologique

dans le domaine de la forme]

Vol. 11, $\mathrm{n}^{\text {os }}$ 2/3: 73 s

Sergueï Eisenstein : l'acteur

manquant

Vol. 11, $\mathrm{n}^{\text {os }} 2 / 3: 199 \sim \mathrm{z}$

Sublimation comme formation de

la forme. Notes sur un article inédit

d'Eisenstein (La)

Vol. 11, $\mathrm{n}^{\text {os }} 2 / 3: 111 \sim \mathrm{z}$

Textes d'Eisenstein cités dans

l'ensemble du numéro

Vol. 11, ${ }^{\text {os }} 2 / 3: 271 \sim \mathrm{z}$

EMMERICH Roland

Independance Day, le jour de la

riposte

Vol. 8, n 3: 9 z

FALARDEAU Pierre

Temps des bouffons (Le)

Vol. 9, n ${ }^{\text {os }}$ 2/3: 205 d

FASSBINDER Rainer Werner

Effi Briest

Vol. 4, n $1: 66 \sim \mathrm{z}$

FINCHER David

Alien 3

Vol. 7, $\mathrm{n}^{\text {os }} 1 / 2: 121 \sim \mathrm{Z}$

FORD John

Italian and Irish Filmmakers in

America : Ford, Capra, Coppola and

Scorsese (Lee Lourdeaux)

Vol. 2, n ${ }^{\circ} 1: 170-1$

\section{FOUCAULT Michel}

Archéologie du cinéma: de l'histoire

à l'épistémologie

Vol. 14, n ${ }^{\text {os }} 2 / 3: 19 \sim d$

FRAPPIER Roger

Dernier glacier (Le)

Vol. 4, $\mathrm{n}^{\circ} 2$ : 125 p 


\section{FREUD Sigmund}

Freud et l'" intuition

cinégraphique»: psychanalyse,

cinéma et épistémologie

Vol. 14, $\mathrm{n}^{\text {os }} 2 / 3: 53 \sim \mathrm{d}$

\section{GERVAIS Suzanne}

Climats

Vol. 13, $\mathrm{n}^{\text {os }} 1 / 2: 157 \sim \mathrm{z}$

Cycle

Vol. 13, n ${ }^{\text {os }} 1 / 2: 156 \sim \mathrm{z}$

GILLIAM Terry

Armée des douze singes (L')

Vol. 13, n 3: 53 z

GIRARD François

Cargo

Vol. 8, $\mathrm{n}^{\text {os }} 1 / 2: 35 \sim \mathrm{z}$

GODARD Jean-Luc

À bout de souffle

Vol. 2, n ${ }^{\text {os }} 2 / 3$ : 57 d

Accords et faux raccords entre les

conceptions du cinéma de Jean-Luc

Godard et de Gilles Deleuze

Vol. 13, n 3: 173 d

Allemagne neuf zéro

Vol. 4, n' 3: 73 z

Deux ou trois choses que je sais

d'elle

Vol. 10, $\mathrm{n}^{\text {os }} 2 / 3: 65 \sim \mathrm{d}$

Histoire(s) du cinéma

Vol. $8, \mathrm{n}^{\text {os }} 1 / 2: 125 \sim \mathrm{z}$

Vol. 13, n $3: 9 \sim$ z

Pierrot le fou

Vol. 10, $\mathrm{n}^{\text {os }} 2 / 3$ : 65 d

GODBOUT Jacques

Alias Will James

Vol. $1, n^{\text {os }} 1 / 2$ : $94 \sim p$

GREENAWAY Peter

Prospero's Books

Vol. 10, $n^{\text {os }} 2 / 3: 43 \sim \mathrm{z}$

GRÜNDGENS Gustaf

Schritt vom Wege (Der)

Vol. 4, n $1: 66 \sim \mathrm{z}$

\section{GUTIÉRREZ ALEA Tomas}

Mémoires du sous-développement

Vol. 8, n 3: 105 z
HAWKS Howard

Impossible monsieur Bébé (L')

Vol. 13, $\mathrm{n}^{\text {os }} 1 / 2: 176 \sim \mathrm{d}$

HÉBERT Pierre

Musicalité ou oralité? (Réflexions

d'un cinéaste qui voulait «faire

comme un musicien »)

Vol. 3, n $1:$ 43 s

HERZOG Werner

Certains paysages d'Herzog sous la

loupe du système des attractions

Vol. 12, $\mathrm{n}^{\circ} 1: 87 \sim \mathrm{z}$

HOU Hsiao-hsien

Un temps pour vivre, un temps pour mourir

Vol. 3, n ${ }^{\text {s }} 2 / 3: 28 \sim \mathrm{p}$

HUANG Jianzhong

Affaire du canon noir (L')

Vol. 3, $\mathrm{n}^{\text {os }}$ 2/3: 39 p

Samsara

Vol. 3, n ${ }^{\text {os }}$ 2/3: 39 p

IVENS Joris

Chant des fleuves (Le)

Vol. 12, n 3: 109 z

JUGERT Rudolf

Rosen im Herbst

Vol. 4, n $1: 60 \sim \mathrm{z}$

JUTRA Claude

À tout prendre

Vol. 7, $\mathrm{n}^{\text {os }} 1 / 2$ : 44 d

Mon oncle Antoine

Vol. 7, $\mathrm{n}^{\circ}$ 3: 161 t

\section{KAEL Pauline}

"Common People with Common

Feelings" : Pauline Kael, James Agee, and the Public Sphere of

Popular Film Criticism

Vol. 6, n ${ }^{\text {os }}$ 2/3 : 113 d

KASSOVITZ Mathieu

Haine (La)

Vol. 10, $\mathrm{n}^{\text {os }} 2 / 3: 65 \sim \mathrm{d}$

Vol. 12, $\mathrm{n}^{\circ} 1: 71 \sim \mathrm{z}$ 
KEATON Buster

Corps au cinéma: Keaton, Bresson, Cassavetes (Le) (Vincent Amiel)

Vol. 11, n ${ }^{\text {os }}$ 2/3: 309 1

KOUYATÉ Dani

Keita! l'héritage du griot

Vol. 11, n $1: 45 \sim \mathrm{z}$

KUBRICK Stanley

Barry Lyndon

Vol. 4, $\mathrm{n}^{\circ} 1: 23 \sim \mathrm{z}$

Kubrick as Architect

Vol. 9, $\mathrm{n}^{\circ} 1: 117-\mathrm{z}$

Shining

Vol. 4, $\mathrm{n}^{\circ} 1: 115 \sim \mathrm{z}$

Stanley Kubrick. A Narrative and

Stylistic Analysis (Mario Falsetto)

Vol. 6, $n^{\text {os }} 2 / 3: 217-1$

Vol. 13, $n^{\text {os }} 1 / 2: 229-1$

KWAN Stanley

Rouge

Vol. 3, $\mathrm{n}^{\text {os }} 2 / 3: 157 \sim \mathrm{p}$

LAFOND Jean-Daniel

Voyage au bout de la route ou La

ballade du pays qui attend (Le)

Vol. 1, $\mathrm{n}^{\text {os }} 1 / 2$ : 95 $\sim \mathrm{p}$

Vol. $1, n^{\text {os }} 1 / 2: 108 \sim \mathrm{p}$

LAMBERT Lucie

Avant le jour

Vol. 12, n 1 : 135 p

Paysage sous les paupières

Vol. 12, n ${ }^{\circ} 1: 134 \sim \mathrm{p}$

LANCTÔT Micheline

Poursuite du bonheur (La)

Vol. 1, $\mathrm{n}^{\text {os }} 1 / 2$ : 92 p

Sonatine

Vol. 7, $\mathrm{n}^{\circ} 3: 171 \sim \mathrm{t}$

Vol. $8, \mathrm{n}^{\text {os }} 1 / 2: 187 \sim \mathrm{z}$

LANG Fritz

Fritz Lang en Amérique (Peter

Bogdanovich)

Vol. 9, $\mathrm{n}^{\text {os }}$ 2/3: 238 1

Fritz Lang (Lotte Eisner)

Vol. 9, $\mathrm{n}^{\text {os }} 2 / 3: 237 \sim 1$
Fritz Lang: The Nature of the

Beast. A Biography (Patrick

McGilligan)

Vol. 9, $\mathrm{n}^{\text {os }} 2 / 3: 240 \sim 1$

Fritz Lang. Trois lumières (Alfred

Abel)

Vol. 9, $\mathrm{n}^{\text {os }}$ 2/3: 236 1

Metropolis

Vol. 8, n 3: 31 z

Montagne des superstitions et autres histoires (La) (Cornelius Schnauber) Vol. 9, $\mathrm{n}^{\text {os }} 2 / 3: 239-1$

Mort d'un carriériste et autres histoires (Cornelius Schnauber) Vol. 9, $\mathrm{n}^{\text {os }} 2 / 3$ : 239 -1

Tout ce que vous avez toujours voulu savoir sur Fritz Lang, sans jamais

oser le demander

Vol. 9, $\mathrm{n}^{\text {os }} 2 / 3: 235 \sim \mathrm{z}$

LANGLOIS Michel

Cap Tourmente

Vol. 9, n $^{\text {os }}$ 2/3: 85 z

LANZMANN Claude

Shoah

Vol. 12, n ${ }^{\circ} 1: 147 \sim \mathrm{c}$

LAUZON Jean-Claude

Léolo

Vol. 6, n ${ }^{\text {os }} 2 / 3: 84 \sim \mathrm{d}$

Vol. $8, \mathrm{n}^{\text {os }} 1 / 2: 35 \sim \mathrm{z}$

Vol. 9, $\mathrm{n}^{\text {os }}$ 2/3: 175 $\mathrm{z}$

LEDUC Jacques

Dernier glacier (Le)

Vol. 4, n 2 : 125 - p

On est loin du soleil

Vol. 9, n ${ }^{\text {os }}$ 2/3 : 209 d

LUDERER Wolfgang

Effi Briest

Vol. 4, $\mathrm{n}^{\circ} 1: 59 \sim \mathrm{z}$

LUMIÈRE (Les frères)

Le paysage dans les vues Lumière

Vol. 12, $\mathrm{n}^{\circ} 1: 15 \sim \mathrm{z}$

LVOFF John

Salle de bain (La)

Vol. 4, n $1: 103 \sim \mathrm{z}$ 
LYNCH David

Lost Highway

Vol. 13, n 3: 95 z

LYNN Jonathan

Clue

Vol. 10, nº 1: 99 d

McDONALD Bruce

Roadkill

Vol. 3, n 1 : 132 p

McLAREN Norman

Blinkity Blank

Vol. 14, $\mathrm{n}^{\text {os }}$ 2/3: 127 -d

Pas de deux

Vol. 9, n ${ }^{\text {os }}$ 2/3 : 225 d

MALLE Louis

Au revoir les enfants

Vol. 9, $n^{\text {os }}$ 2/3: 21 z

Humain, trop humain

Vol. 9, $\mathrm{n}^{\text {os }}$ 2/3 : 203 d

Zazie dans le métro

Vol. 4, n 3: 135 z

MARKER Chris

Jetée (La)

Vol. 13, n 3: 53 z

Sans soleil

Vol. 13, n 3: 33 z

METTLER Peter

Eastern Avenue

Vol. 3, n ${ }^{\circ} 1: 37 \sim \mathrm{z}$

Scissere

Vol. 3, n $1: 36 \sim \mathrm{z}$

Top of His Head (The)

Vol. 3, n ${ }^{\circ} 1: 38 \sim \mathrm{z}$

MITTERRAND Frédéric

Lettres d'amour en Somalie

Vol. 4, n ${ }^{\circ} 2: 83 \sim \mathrm{z}$

MURNAU Friedrich Wilhelm

Tartuffe

Vol. 4, n ${ }^{\circ} 1: 35 \sim \mathrm{d}$

MONROE Marilyn

The Subway Grate Scene in The

Seven Year Itch: "The Staging of an

Appearance-As-Disappearance"

Vol. $14, n^{\text {os }} 2 / 3: 213 \sim \mathrm{z}$
NIMOY Leonard

Trois hommes et un bébé

Vol. 1, $\mathrm{n}^{\text {os }} 1 / 2: 115 \sim \mathrm{z}$

NOËL Jean-Guy

Tinamer

Vol. 2, $\mathrm{n}^{\text {os }}$ 2/3: 209 z

PASOLINI Pier Paolo

Résistance du corps dans l'image cinématographique. La mort, le

mythe et la sexualité dans le cinéma

de Pasolini (La)

Vol. 7, $\mathrm{n}^{\text {os }} 1 / 2: 89 \sim \mathrm{z}$

St. Paul: The Unmade Movie

Vol. 9, n ${ }^{\text {os }}$ 2/3: 67 z

PASTRONE Giovanni

Cabiria

Vol. 10, n $1: 55 \sim \mathrm{z}$

Vol. 10, $n^{\text {os }} 2 / 3$ : 207 d

PENG Xiaolian

Une histoire de femmes

Vol. 3, n ${ }^{\text {os }}$ 2/3: 137 p

PERRAULT Pierre

Oumigmag (L')

Vol. 5, n ${ }^{\circ}$ 3: $101 \sim \mathrm{z}$

Vol. 5, n' 3: 115 z

Paysage dans la tradition

documentaire québécoise: un

regard off sur la parole (Le)

Vol. 12, $\mathrm{n}^{\circ} 1:$ 127 z

Pour la suite du monde

Vol. 7, n 3 : 164 t

POOL Léa

Femme de l'hôtel (La)

Vol. 3, n 1 : 127 p

Vol. 8, $\mathrm{n}^{\text {os }} 1 / 2: 35 \sim \mathrm{z}$

Strass Café

Vol. 6, $\mathrm{n}^{\text {os }}$ 2/3 : 199 d

PORTER Edwin $S$.

Attaque du grand rapide (L')

Vol. 5, no 3: 9 d

POTTER Sally

Orlando

Vol. 13, $\mathrm{n}^{\text {os }} 1 / 2$ : 177 d 


\section{RAY Nicholas}

Nick's Movie

Vol. 4, n ${ }^{\circ}$ 2: 101 z

\section{REICH Steve}

Phase Patterns

Vol. 9, n ${ }^{\text {os }} 2 / 3: 227 \sim d$

\section{RENOIR Jean}

Boudu sauvé des eaux

Vol. 10, $\mathrm{n}^{\text {os }} 2 / 3: 65 \sim \mathrm{d}$

Chienne (La)

Vol. 3, n $1: 19 \sim \mathrm{z}$

French cancan

Vol. 12, $\mathrm{n}^{\circ}$ 3: 11 z

Jean Renoir à Hollywood ou La

recherche américaine d'une image

française

Vol. $1, \mathrm{n}^{\text {os }} 1 / 2: 73 \sim \mathrm{z}$

Journal d'une femme de chambre

(Le)

Vol. $1, \mathrm{n}^{\text {os }} 1 / 2: 81 \sim \mathrm{z}$

Vivre libre

Vol. 1, n ${ }^{\text {os }} 1 / 2: 77 \sim \mathrm{z}$

RESNAIS Alain

Alain Resnais. Une lecture

topologique (Sarah Leperchey)

Vol. 14, $\mathrm{n}^{\text {os }} 2 / 3: 247$ -

Hiroshima, mon amour

Vol. 5, $\mathrm{n}^{\text {os }} 1 / 2: 135 \sim \mathrm{z}$

Vol. 9, n ${ }^{\text {os }} 2 / 3: 159 \sim \mathrm{z}$

Itinéraire d'Alain Resnais (L')

(René Prédal)

Vol. 9, nº 1: 159 -1

Stavisky

Vol. 5, n ${ }^{\text {os }} 1 / 2: 27 \sim \mathrm{d}$

RIMMER David

Fracture

Vol. 9, $\mathrm{n}^{\text {os }} 2 / 3: 225 \sim \mathrm{d}$

RIVETTE Jacques

Belle noiseuse $(\mathrm{La})$

Vol. 4, $\mathrm{n}^{\circ} 1: 73 \sim \mathrm{z}$

ROBESON Paul

Utopian Visions in Cold War

Documentary: Joris Ivens, Paul

Robeson and Song of the Rivers (1954)

Vol. 12, n' 3: 109 z
ROHMER Éric

Éric Rohmer, Six contes moraux

Vol. 9, $\mathrm{n}^{\text {os }} 2 / 3: 28 \sim \mathrm{z}$

\section{ROSSELLINI Roberto}

Stromboli

Vol. 13, n ${ }^{\text {os }} 1 / 2: 129 \sim \mathrm{z}$

\section{ROZEMA Patricia}

White Room

Vol. 3, $\mathrm{n}^{\circ} 1:$ 137 p

SANDRICH Mark

Danseur du dessus (Le)

Vol. 5, $\mathrm{n}^{\text {os }} 1 / 2: 211 \sim \mathrm{z}$

Joyeuse divorcée (La)

Vol. 7, $\mathrm{n}^{\text {os }} 1 / 2: 151 \sim \mathrm{z}$

SARTRE Jean-Paul

Montage, simultanéité et continuité

dans Le Sursis de Sartre

Vol. 8, n ${ }^{\circ}$ 3: 75 z

\section{SCORSESE Martin}

Italian and Irish Filmmakers in

America: Ford, Capra, Coppola and

Scorsese (Lee Lourdeaux)

Vol. 2, $\mathrm{n}^{\circ} 1: 170 \sim 1$

SCOTT Ridley

Alien, le huitième passager

Vol. $7, \mathrm{n}^{\text {os }} 1 / 2: 121 \sim \mathrm{z}$

SERREAU Coline

Trois hommes et un couffin

Vol. 1, $\mathrm{n}^{\text {os }} 1 / 2: 115 \sim \mathrm{z}$

SHAW William

Vol. 6, $\mathrm{n}^{\circ} 1: 47 \sim \mathrm{z}$

SIMONEAU Yves

Fous de Bassan (Les)

Vol. 9, $\mathrm{n}^{\text {os }} 2 / 3$ : 103 z

Pouvoir intime

Vol. 2, n $1: 67$ d

SPIELBERG Steven

Couleur pourpre (La)

Vol. 13, n ${ }^{\text {os }} 1 / 2: 180 \sim d$

Jurassic Park

Vol. 4, $\mathrm{n}^{\circ} 3: 33-\mathrm{t}$

Liste de Schindler (La)

Vol. 7, n ${ }^{\text {os }} 1 / 2: 21 \sim \mathrm{z}$ 
SVANKMAJER Jan

Dark Alchemy. The Films of Jan

Svankmajer (Peter Hames)

Vol. 8, $\mathrm{n}^{\text {os }} 1 / 2: 215-1$

TACCHELLA Jean-Charles

Travelling avant

Vol. 9, n ${ }^{\text {os }} 2 / 3: 214 \sim d$

TARKOVSKI Andrei

Miroir (Le)

Vol. 13, no 3: 119 z

Tarkovski, cinéaste cynique

Vol. 4, n 3 : 15 z

TIAN Zhuangzhuang

Eunuque impérial (L')

Vol. 3, $\mathrm{n}^{\text {os }}$ 2/3 : 39 p

TOURNEUR Jacques

Féline (La)

Vol. 5, n' 3: 29 d

TRUFFAUT François

Aventure américaine de l'œuvre de

François Truffaut (L') (Laurence

Alfonsi)

Vol. 12, n 3 : 175 1

Dernier métro (Le)

Vol. 7, $\mathrm{n}^{\text {os }} 1 / 2: 205 \sim \mathrm{z}$

Vol. 9, n ${ }^{\text {os }} 2 / 3: 212 \sim d$

Femme d'à côté (La)

Vol. $7, \mathrm{n}^{\text {os }} 1 / 2: 205 \sim \mathrm{z}$

François Truffaut et Antoine Doinel

Vol. 9, $\mathrm{n}^{\text {os }}$ 2/3: 24 $\mathrm{z}$

Lectures asiatiques de l'œuvre de

François Truffaut (Laurence

Alfonsi)

Vol. 12, n 3 : 175 1

VERHOEVEN Paul

Basic Instinct

Vol. 3, $\mathrm{n}^{\text {os }} 2 / 3: 223 \sim \mathrm{z}$

VISCONTI Luchino

En deçà et au-delà du cinéma

moderne. Visconti, mélancolie et néo-baroque

Vol. $8, \mathrm{n}^{\text {os }} 1 / 2: 113 \sim \mathrm{z}$
Le corps à corps des images dans

l'œuvre de Visconti

Vol. 7, $\mathrm{n}^{\text {os }} 1 / 2: 109 \sim \mathrm{z}$

Senso

Vol. 3, n $1: 25 \sim \mathrm{z}$

WENDERS Wim

Ailes du désir (Les)

Vol. 1, n 3 : 129 d

Vol. 5, $\mathrm{n}^{\text {os }} 1 / 2$ : 105 z

Nick's Movie

Vol. 4, n 2 : 101 z

WIDRICH Virgil

Fast Film

Vol. 14, n ${ }^{\text {os }} 2 / 3$ : 131 d

WILDER Billy

Boulevard du Crépuscule

Vol. 5, no 3: 65 - z

Seven Year Itch (The)

Vol. 14, $n^{\text {os }}$ 2/3: 213 z

WISE Robert

Maison du diable (La)

Vol. 5, n ${ }^{\circ} 3$ : 52 d

XIE Fei

Année de mon signe (L')

Vol. 3, n ${ }^{\text {os }} 2 / 3$ : 39 p

ZEMECKIS Robert

Qui veut la peau de Roger Rabbit?

Vol. 5, $\mathrm{n}^{\circ}$ 3: 151 z

\section{ZHANG Yimou}

Deux pôles yang du nouveau

cinéma chinois: Chen Kaige et

Zhang Yimou

Vol. 3, $\mathrm{n}^{\mathrm{os}}$ 2/3: 103 p

Ju dou — Le sang du père

Vol. 3, $\mathrm{n}^{\text {os }}$ 2/3: 61 z

Sorgho rouge (Le)

Vol. 3, n ${ }^{\text {os }} 2 / 3: 61 \sim \mathrm{z}$ 